\title{
Cardiac Surgery in Germany during 2013: A Report on Behalf of the German Society for Thoracic and Cardiovascular Surgery
}
A. Funkat ${ }^{1}$
A. Beckmann²
J. Lewandowski
M. Frie $^{3}$
M. Ernst ${ }^{4}$
W. Schiller ${ }^{5}$
J. F. Gummert ${ }^{6}$
J. Cremer $^{4}$

${ }^{1}$ Department of Cardiac Surgery, University of Leipzig, Leipzig, Germany

${ }^{2}$ German Society for Thoracic and Cardiovascular Surgery,

Langenbeck-Virchow-Haus, Berlin, Germany

${ }^{3}$ FOM Hochschule fuer Oekonomie and Management, Essen, Germany

${ }^{4}$ Department of Thoracic and Cardiovascular Surgery, University Kiel, Kiel, Germany

${ }^{5}$ Department of Cardiac Surgery, University Bonn, Bonn, Germany

${ }^{6}$ Clinic for Thoracic and Cardiovascular Surgery, Heart and Diabetes

Center NRW, Bad Oeynhausen, Germany

Thorac Cardiovasc Surg 2014;62:380-392.

Address for correspondence Anne-K. Funkat, Dr.-Ing, Klinik für Herzchirurgie, Herzzentrum Leipzig GmbH - Universitätsklinik, Strümpellstr. 39, 04289 Leipzig, Germany (e-mail: funkat.ch@herzzentrum-leipzig.de).

\begin{abstract}
Keywords

- cardiac surgery

- outcome

- registry

- congenital heart disease

- aortic surgery

- organ transplantation

On the basis of a voluntary registry of the German Society for Thoracic and Cardiovascular Surgery (GSTCVS), data of all cardiac surgical procedures performed in 79 German cardiac surgical units during the year 2013 are presented. In 2013, a total of 99,128 cardiac surgical procedures (implantable cardioverter defibrillator $[I C D]$ and pacemaker procedures excluded) were submitted to the registry. More than $13.8 \%$ of the patients were older than 80 years, which remains equal in comparison to the previous year. In-hospital mortality in 40,410 isolated coronary artery bypass grafting procedures ( $84.5 \%$ on-pump and $15.5 \%$ offpump) was $2.9 \%$. In 29,672 isolated valve procedures (including 7,722 catheter-based procedures), an in-hospital mortality of $4.7 \%$ was observed. This long-lasting registry of the GSTCVS will continue to be an important tool for quality control and voluntary public reporting by illustrating current facts and developments of cardiac surgery in Germany.
\end{abstract}

\section{Introduction}

Increasing demands for quality assurance in medicine-by patients, relatives, insurance companies, and authorities all over the world-have stimulated the development of a wide range of registries and other tools to answer those needs. As early as in 1978, the board of directors of the German Society for Thoracic and Cardiovascular Surgery (GSTCVS) (www. dgthg.de) decided to set up an annually updated database of all cardiac surgical procedures in terms of a voluntary registry. Since 1989, the updated data of the registry are published annually. ${ }^{1-24}$ The aim of this registry continues to illustrate developments and trends in cardiac surgery in Germany, and it enables each participating cardiac surgical unit to compare its own results with the nationwide achievements.

received

April 7, 2014

accepted

April 15, 2014

published online

July 4, 2014

For monitoring actual conditions as well as the development in cardiac medicine, the registry includes particular techniques such as off-pump cardiac surgery or minimal invasive mitral valve operations and also innovative technologies such as transapical or transvascular aortic valve implantation (TAVI). Thereby important findings for present patient safety and the future of patient care may be collected and evaluated.

The data presented in this report comprehend assorted data of the year 2013.

\section{Materials and Methods}

Since 2004, a standardized questionnaire gathers detailed information about each individual procedure exactly defined

(c) 2014 Georg Thieme Verlag KG Stuttgart · New York
DOI http://dx.doi.org/ 10.1055/s-0034-1383430. ISSN 0171-6425. 
by a German adaption of the International Classification of Procedures in Medicine called operation code (Operationenund Prozedurenschlüssel).

All participants were requested to complete the structured questionnaire until January 20, 2014, asking for all performed procedures and associated in-hospital mortality. The recommended path for data export is an electronic transmission of an encrypted file which had to be addressed to the office of the GSTCVS. After transaction, the data were decrypted, evaluated for completeness, and compiled for further analysis, thus ensuring anonymity for each individual institution. This compilation algorithm guarantees a high compliance for submission of the complete dataset.

Inclusion criteria for the registry 2013 were all patients receiving cardiac surgical procedures performed between January 1, 2013, and December 31, 2013, unrelated to the date of admission or discharge as compared with other registries. Alike to all previous years, the number of procedures was counted rather than individual patients. For example, if a patient required additional coronary artery bypass grafting (CABG) due to a complication after initial aortic valve replacement during one admission, one count in the category "aortic valve replacement" and another in the category "coronary surgery" are enumerated. Thus, the registry contains more procedures than the real number of patients operated on.

Death of patients was defined as in-hospital mortality. As per the definition, the observed mortality is always attributed to the first cardiac procedure, for example, the death of a patient requiring CABG due to a complication of an aortic valve procedure would only be attributed to the aortic valve procedure.

The main reason for this structural setup of the registryestablished over several decades-is to keep the German data privacy act with its specific regulations for patients. Furthermore, it seemed to be relevant getting detailed information about all performed procedures and not only the number of treated patients. Last but not the least, the process of data acquisition had to be simplified for all cardiac surgery units in Germany thus enabling the submission of a complete dataset, regardless of the locally existing hard- and software used for data management.

In 2013, a total of 79 institutions performed heart surgery. Fortunately, all units answered the questionnaire and delivered a complete dataset for the year 2013 including hospital mortality rates.

\section{Registry Data 2013}

-Table 1 illustrates the development of procedures using extracorporeal circulation (ECC) over one decade in Germany. Since 2010, the count of heart operations using ECC remains on a stable level.

Overall, 180,367 procedures were reported to the registry for the year 2013, an increase of 1.5\% (2012: 177,694 procedures). A total of 102,845 cardiac surgical procedures (excluded: implantable cardioverter defibrillator (ICD), pacemakers, and miscellaneous procedures without ECC) display an increase of $0.94 \%(n=958)$ compared with the year 2012 (101,887 procedures) (-Table 2 ). - Tables 3 to $\mathbf{6 , ~ V 1}$ to V7, C1 to C3, Con1 and Con2, Mis1 to Mis5, and - Figs. 1 to 9 demonstrate the compiled registry data of 2013 for various categories.

Compared with the data of previous years, several important developments continued in 2013 almost unchanged. Over the past 10 years, the distribution of patient age (-Fig. 6) showed a shift to older patients with presently $54.0 \%$ of the cardiac procedures performed in patients of at least 70 years of age and $13.8 \%$ in patients of 80 years or older. However, mortality remained on the same low level or even decreased slightly over the represented decade (see - Fig. 2). The rate of CABG decreased over the past years, while the relative number of off-pump CABG showed a slight increase to 15.5\% (2012: 15.4\%) (-Fig. 3).

Since 2004 , more than $50 \%$ of isolated mitral valve procedures are reconstructions, in 2013 mitral valve reconstruction could be achieved in $65.8 \%$ of the procedures (-Fig. 8). On the basis of the fact that all isolated mitral valve procedures are included, regardless of the underlying disease, valve morphology, or urgency of operation, it has to be assumed that the relative rate of mitral valve reconstruction would certainly be even higher if patients without possibility or indication for reconstruction would have been excluded (e.g., mitral valve stenosis, calcifications, or endocarditis). In other publications, for example, Gammie et $\mathrm{al}^{25}$ patients with mitral valve stenosis, endocarditis, and emergency procedures were excluded. Therefore, the published rate of mitral valve repair must be interpreted with caution compared with this registry.

The continued increase of left ventricular assist device implantations ( - Fig. 10) emphasizes the increasing relevance of mechanical circulatory support.

Again the most remarkable trend is the repeated extensive increase of TAVI procedures (-Fig. 5), although the number of

Table 1 Open heart procedures under use of ECC in Germany from 2004 to 2013

\begin{tabular}{|l|l|l|l|l|l|l|l|l|l|l|}
\hline & 2004 & 2005 & 2006 & 2007 & 2008 & 2009 & 2010 & 2011 & 2012 & 2013 \\
\hline Units & 79 & 79 & 80 & 80 & 79 & 80 & 79 & 78 & 79 & 79 \\
\hline Operations & 96,340 & 91,967 & 91,057 & 91,618 & 89,773 & 86,916 & 84,686 & 84,402 & 84,388 & 84,040 \\
\hline Average per unit & 1,219 & 1,164 & 1,138 & 1,145 & 1,136 & 1,086 & 1,072 & 1,082 & 1,068 & 1,064 \\
\hline
\end{tabular}

Abbreviation: ECC, extracorporeal circulation. 
382 Cardiac Surgery in Germany during 2013 Funkat et al.

Table 2 Cardiac surgery categories 2013 compared with 2012

\begin{tabular}{|l|l|l|l|l|}
\hline & With ECC & Without ECC & Total & \% change \\
\hline Heart valve procedures & 22,119 & 7,553 & 29,672 & +4.0 \\
\hline Coronary artery bypass grafting & 47,582 & 6,680 & 54,262 & -1.9 \\
\hline Surgery for CHD & 4,721 & 855 & 5,576 & 7,671 \\
\hline Surgery of thoracic aorta & 7,045 & 626 & 2,619 & -0.3 \\
\hline Cardiac surgery other & 1,394 & 1,225 & 2,657 & +3.9 \\
\hline Assist device procedures & 791 & 1,866 & 25,738 & +5.9 \\
\hline Pacemaker and ICD procedures & 59 & 25,679 & 52,172 & +1.5 \\
\hline Extracardiac surgery & 329 & 51,843 & 180,367 & +2.0 \\
\hline Total & 84,040 & 96,327 & 3.2 & +1.5 \\
\hline
\end{tabular}

Abbreviations: CHD, congenital heart disease; ECC, extracorporeal circulation.

Table 3 Units assorted by volume categories (procedures with or without ECC)

\begin{tabular}{|l|l|l|l|l|l|}
\hline Procedure volume & $<500$ & $500-999$ & $1,000-1,499$ & $1,500-1,999$ & $2,000-5,000$ \\
\hline Units & 7 & 25 & 26 & 12 & 9 \\
\hline Min-max & $237-451$ & $557-995$ & $1,028-1,487$ & $1,538-1,987$ & $2,075-3,754$ \\
\hline Average per unit & 355 & 808 & 1,201 & 1,723 & 2,727 \\
\hline
\end{tabular}

Abbreviation: ECC, extracorporeal circulation.

Table 4 Total number of units according to surgical procedures in 2013

\begin{tabular}{|l|l|}
\hline & Units \\
\hline Coronary artery bypass grafting & 78 \\
\hline Heart valve surgery & 78 \\
\hline Surgery for CHD in children $<1$ y with ECC & $24^{\text {a }}$ \\
\hline Heart transplantation ${ }^{\text {b }}$ & $22^{\text {b }}$ \\
\hline Heart-lung transplantation & 4 \\
\hline
\end{tabular}

Abbreviations: CHD, congenital heart disease; ECC, extracorporeal circulation.

$\mathrm{a}_{n}=2,047$; thereof: $2-16$ operations in three units, $26-41$ operations in seven units, 58-98 operations in six units, and 114-318 operations in eight units.

$b_{n}=301 ; 67 \%$ of the heart transplantations (HTx) in 2013 were performed by 7 of 24 units with $\geq 15$ HTx per year; thereof: 1-4 transplantations in eight units, 5-9 transplantations in five units, 10-17 transplantations in six units, and 22-75 transplantations in five units.

Table 5 Additional data for procedures with ECC in 2013 compared with 2012

\begin{tabular}{|l|l|l|l|l|}
\hline & \multicolumn{2}{|c|}{2013} & \multicolumn{2}{c|}{2012} \\
\hline Emergency operations & 11,944 & $11.6 \%$ & 11,878 & $11.6 \%$ \\
\hline Redo procedures & 8,284 & $8.1 \%$ & 8,424 & $8.2 \%$ \\
\hline Age $>69 \mathrm{y}^{\mathrm{a}}$ & 98,042 & $54.0 \%$ & 97,572 & $53.7 \%$ \\
\hline
\end{tabular}

Abbreviation: ECC, extracorporeal circulation.

Note: The numbers in each category reflect procedures and not individual patients.

apatients $<20$ years are excluded. 
Cardiac Surgery in Germany during 2013 Funkat et al. 383

Table 6 Gender distribution

\begin{tabular}{|l|l|l|}
\hline Male/female distribution & Male (\%) & Female (\%) \\
\hline All procedures & 66 & 34 \\
\hline Heart valve procedures & 55 & 45 \\
\hline Coronary artery bypass grafting & 76 & 24 \\
\hline Surgery for CHD & 55 & 45 \\
\hline Surgery of thoracic aorta & 68 & 32 \\
\hline Cardiac surgery other & 48 & 52 \\
\hline Assist device procedures & 73 & 27 \\
\hline Pacemaker and ICD procedures & 64 & 36 \\
\hline Extracardiac surgery & 65 & 35 \\
\hline
\end{tabular}

Abbreviation: $\mathrm{CHD}$, congenital heart disease.

Note: Coronary artery bypass surgery $(47,582$ on-pump and 6,680 offpump procedures) and all procedures for congenital heart surgery are included in this table.

isolated aortic valve replacement procedures remained on a stable level. Starting in 2006 with just 78 implantations ( $0.67 \%$ of isolated aortic valve procedures), in 2013 a total of 7,246 (38.1\%) TAVI were reported to the registry. It must be emphasized that the 79 institutions which contribute their data to this registry do not represent all departments performing TAVI in Germany. In addition, there are some institutions performing TAVI via transvascular access without a cardiac surgery on-site. This proceeding does not correlate to the recommendations of the European guideline on the management of valvular heart disease, version 2012. ${ }^{26}$

In this context, the short-, mid-, and long-term results of the German Aortic Valve Registry ${ }^{27,28}$ and the annual findings of the legal quality assurance ( $\$ 137$ SGB V), conducted by the AQUA Institute, are of outstanding patient benefit.

Table V1 Isolated heart valve procedures

\begin{tabular}{|l|l|l|l|}
\hline Procedures & $N$ & Deaths & $\%$ \\
\hline Single & 18,108 & 622 & 3.4 \\
\hline Double & 3,363 & 285 & 8.5 \\
\hline Triple & 380 & 52 & 13.7 \\
\hline Single transcatheter access & 7,712 & 434 & 5.6 \\
\hline Double transcatheter access & 10 & 1 & 10.0 \\
\hline Unspecified & 99 & 6 & 6.1 \\
\hline Total & 29,672 & 1,400 & 4.7 \\
\hline
\end{tabular}

Notes: Combined procedures (with CABG, aortic surgery) are excluded. Transcatheter valve procedures: 7,246 aortic valve implantations; 58 mitral valve implantations; 404 mitral valve repairs, 3 tricuspidal valve repairs, 10 combined aortic and mitral valve procedures, and 1 pulmonary valve implantation.
Table V2 Access type in single valve procedures

\begin{tabular}{|c|c|c|c|}
\hline Position & $N$ & Deaths & $\%$ \\
\hline \multicolumn{4}{|l|}{ Aortic valve } \\
\hline Sternotomy & 9,541 & 294 & 3.1 \\
\hline Partial sternotomy & 2,350 & 46 & 2.0 \\
\hline Transvascular & 4,360 & 200 & 4.6 \\
\hline Transapical & 2,886 & 219 & 7.6 \\
\hline \multicolumn{4}{|l|}{ Mitral valve } \\
\hline Sternotomy & 3,083 & 199 & 6.5 \\
\hline Minimal invasive & 2,548 & 30 & 1.2 \\
\hline Transcatheter & 462 & 14 & 3.0 \\
\hline \multicolumn{4}{|l|}{ Tricuspidal valve } \\
\hline Sternotomy & 424 & 45 & 10.6 \\
\hline Minimal invasive & 131 & 8 & 6.1 \\
\hline Transcatheter & 3 & 1 & 33.3 \\
\hline \multicolumn{4}{|l|}{ Pulmonary valve } \\
\hline Sternotomy & 31 & 0 & 0.0 \\
\hline Minimal invasive & 0 & - & - \\
\hline Transcatheter & 1 & 0 & 0.0 \\
\hline Total & 25,820 & 1,056 & 4.1 \\
\hline
\end{tabular}

Notes: A total of 2,548 (45.2\%) mitral valve procedures were performed by a minimally invasive access. The number of isolated aortic valve procedures by sternotomy remained on the same level counting 11,906 procedures in 2012 and 11,891 in 2013.

Table V3 Isolated aortic valve procedures

\begin{tabular}{|l|l|l|l|}
\hline & $N$ & Deaths & $\%$ \\
\hline Mechanical prosthesis & 1,506 & 26 & 1.7 \\
\hline Xenograft & 10,049 & 311 & 3.1 \\
\hline Homograft & 210 & 2 & 1.0 \\
\hline Repair & 122 & 1 & 0.8 \\
\hline Total & 11,887 & 340 & 2.9 \\
\hline
\end{tabular}

Notes: A total of 2,349 procedures (19.8\%) were performed via partial sternotomy. Transcatheter procedures and apical aortic conduit procedures were not included.

Table V4 Isolated mitral valve procedures

\begin{tabular}{|l|l|l|l|}
\hline & $\boldsymbol{N}$ & Deaths & $\%$ \\
\hline Mechanical prosthesis & 486 & 36 & 7.4 \\
\hline Xenograft & 1,432 & 133 & 9.3 \\
\hline Homograft & 5 & 0 & 0.0 \\
\hline Repair & 3,708 & 60 & 1.6 \\
\hline Total & 5,631 & 229 & 4.1 \\
\hline
\end{tabular}

Notes: A total of 2,548 procedures (45.2\%) were performed via minimally invasive access. Transcatheter procedures were excluded. 
384 Cardiac Surgery in Germany during 2013 Funkat et al.

Table V5 Multiple heart valve procedures

\begin{tabular}{|l|l|l|l|}
\hline Combinations & N & Deaths & $\%$ \\
\hline Aortic + mitral & 1,420 & 129 & 9.1 \\
\hline Mitral + tricuspid & 1,618 & 140 & 8.7 \\
\hline Aortic + tricuspid & 219 & 13 & 5.9 \\
\hline Tricuspid + pulmonary & 17 & 2 & 11.8 \\
\hline Aortic + pulmonary ${ }^{\mathrm{a}}$ & 89 & 1 & 1.1 \\
\hline Aortic + mitral + tricuspid & 380 & 51 & 13.4 \\
\hline Aortic + mitral + pulmonary & 0 & 0 & - \\
\hline Total & 3,743 & 336 & 9.0 \\
\hline
\end{tabular}

Note: Transcatheter procedures were excluded.

ancluding Ross procedures.

\section{Discussion}

The registry of the GSTCVS enables a comprehensive overview of all cardiac surgical procedures performed in Germany in 2013. The accuracy of this registry is considered to be high due to the implemented compilation algorithm using operation coding as a relevant criterion for reimbursement purposes. This is supported by other authors who could demonstrate a high accuracy for major outcome parameters in unaudited registries. ${ }^{29}$ In continuation to previous years, it can be concluded that cardiac surgery in Germany is performed on a constantly high level with a low in-hospital mortality compared with other international registries. This conclusion is especially important in an era of demographic change of the German population resulting in a continuous

Table V6 Mitral valve surgery-implantation/replacement versus repair

\begin{tabular}{|c|c|c|c|c|c|c|c|c|c|c|}
\hline \multirow[t]{2}{*}{ Mitral valve surgery } & \multirow[t]{2}{*}{$N$} & \multirow{2}{*}{$\begin{array}{l}\text { Total } \\
\text { deaths }\end{array}$} & \multirow[t]{2}{*}{$\%$ death } & \multirow[t]{2}{*}{ \% repair } & \multicolumn{3}{|c|}{ Replacement } & \multicolumn{3}{|c|}{ Repair } \\
\hline & & & & & $N$ & Deaths & $\%$ death & $N$ & Deaths & $\%$ death \\
\hline Isolated & 5,631 & 229 & 4.1 & 65.8 & 1,923 & 169 & 8.8 & 3,708 & 60 & 1.6 \\
\hline \multicolumn{11}{|l|}{ Mitral valve + } \\
\hline Aortic valve & 1,420 & 129 & 9.1 & 46.8 & 756 & 99 & 13.1 & 664 & 0 & 0.0 \\
\hline Tricuspid valve repair ${ }^{\mathrm{a}}$ & 1,559 & 127 & 8.1 & 63.4 & 571 & 1 & 0.2 & 988 & 87 & 8.8 \\
\hline CABG & 2,647 & 239 & 9.0 & 71.4 & 756 & 100 & 13.2 & 1,891 & 139 & 7.4 \\
\hline $\begin{array}{l}\text { CABG + aortic } \\
\text { valve replacement }\end{array}$ & 661 & 112 & 16.9 & 58.5 & 274 & 56 & 20.4 & 387 & 56 & 14.5 \\
\hline Total & 11,918 & 836 & 7.0 & 64.1 & 4,280 & 425 & 9.9 & 7,638 & 342 & 4.5 \\
\hline
\end{tabular}

Abbreviation: $\mathrm{CABG}$, coronary artery bypass grafting.

${ }^{\mathrm{a}}$ Fifty-nine procedures (not specified mitral valve + tricuspid valve surgery) were excluded. Deaths: 22.0 (13/59).

Table V7 Transcatheter heart valve procedures

\begin{tabular}{|c|c|c|c|c|c|c|c|}
\hline & \multirow[t]{2}{*}{ Total } & \multirow[t]{2}{*}{ Deaths } & \multirow[t]{2}{*}{$\%$ death } & \multicolumn{2}{|c|}{ With ECC } & \multicolumn{2}{|c|}{ Without ECC } \\
\hline & & & & $N$ & Deaths & $N$ & Deaths \\
\hline $\begin{array}{l}\text { Aortic valve } \\
\text { implantation }\end{array}$ & 7,246 & 419 & 5.8 & 161 & 66 & 7,085 & 353 \\
\hline Transvascular ${ }^{\mathrm{a}}$ & 4,360 & 200 & 4.6 & 69 & 31 & 4,291 & 169 \\
\hline Transapical & 2,886 & 219 & 7.6 & 92 & 35 & 2,794 & 184 \\
\hline Mitral valve & 462 & 14 & 3.0 & 8 & 2 & 454 & 12 \\
\hline Repair & 404 & 13 & 3.2 & 4 & 1 & 400 & 12 \\
\hline Implantation $^{\mathrm{b}}$ & 58 & 1 & 1.7 & 4 & 1 & 54 & 0 \\
\hline $\begin{array}{l}\text { Tricuspidal } \\
\text { valve repair }\end{array}$ & 3 & 1 & 33.3 & 0 & 0 & 3 & 1 \\
\hline $\begin{array}{l}\text { Aortic }+ \\
\text { mitral } \\
\text { valve } \\
\text { implantation }\end{array}$ & 10 & 1 & 10.0 & 0 & 0 & 10 & 1 \\
\hline $\begin{array}{l}\text { Aortic valve } \\
\text { implantation }{ }^{\mathrm{b}}+ \\
\text { CABG }\end{array}$ & 62 & 18 & 29.0 & 24 & 12 & 38 & 6 \\
\hline Total & 7,783 & 453 & 5.8 & 193 & 80 & 7,590 & 373 \\
\hline
\end{tabular}

Abbreviations: CABG, coronary artery bypass grafting; ECC, extracorporeal circulation; TAVI, transapical or transvascular aortic valve implantation. Notes: Pulmonary valve implantation for the correction of congenital lesions are excluded, one procedure was reported for adults without congenital lesion; $39.8 \%$ of TAVI procedures were performed by transapical access, $2.2 \%$ of TAVI procedures were performed under the use of ECC. It has to be assumed that ECC was mostly used in emergency situations, which explains the mortality of $41.0 \%$ in this group. Nevertheless, this underlines the necessity of a cardiac surgery on-site for TAVI procedures.

${ }^{\mathrm{a}}$ Femoral, subclavian, or transaortic access.

${ }^{\mathrm{b}}$ Transvascular and transapical access. 
Table C1 Isolated CABG with ECC and combined procedures with ECC

\begin{tabular}{|c|c|c|c|}
\hline Procedures & $N$ & Deaths & $\%$ \\
\hline CABG & 40,410 & 1,187 & 2.9 \\
\hline \multicolumn{4}{|l|}{ CABG +} \\
\hline TMLR & 3 & 1 & 33.3 \\
\hline Aneurysm resection & 187 & 9 & 4.8 \\
\hline Aortic valve replacement & 7,893 & 371 & 4.7 \\
\hline $\begin{array}{l}\text { Transcatheter aortic valve } \\
\text { implantation }\end{array}$ & 62 & 18 & 29.0 \\
\hline Mitral valve replacement & 756 & 100 & 13.2 \\
\hline Mitral valve repair & 1,891 & 139 & 7.4 \\
\hline $\begin{array}{l}\text { Aortic }+ \text { mitral valve } \\
\text { replacement }\end{array}$ & 274 & 56 & 20.4 \\
\hline $\begin{array}{l}\text { Aortic valve replacement }+ \\
\text { mitral valve repair }\end{array}$ & 387 & 56 & 14.5 \\
\hline Other & 2,398 & 134 & 5.6 \\
\hline Total & 54,261 & 2,071 & 3.8 \\
\hline
\end{tabular}

Abbreviations: CABG, coronary artery bypass grafting; ECC, extracorporeal circulation; TMLR, transmyocardial laser revascularization.

increase of patient age and related comorbidities, both leading to a higher preoperative risk profile.

Compared with 2012, the number of cardiac surgery procedures nearly remains on the same level due to the still increasing number of catheter-based valve procedures.

Table C2 Isolated CABG with ECC

\begin{tabular}{|l|l|l|l|}
\hline Distal anastomosis & $N$ & Deaths & $\%$ \\
\hline Single & 1,078 & 64 & 5.9 \\
\hline Double & 7,113 & 260 & 3.7 \\
\hline Triple & 14,977 & 480 & 3.2 \\
\hline Quadruple & 8,247 & 186 & 2.3 \\
\hline Quintuple + more & 2,742 & 77 & 2.8 \\
\hline Total & 34,157 & 1,067 & 3.1 \\
\hline
\end{tabular}

Abbreviations: CABG, coronary artery bypass grafting; ECC, extracorporeal circulation.

Table C3 Isolated CABG off-pump

\begin{tabular}{|l|l|l|l|}
\hline Distal anastomosis & $N$ & Deaths & $\%$ \\
\hline Single & 1,400 & 37 & 2.6 \\
\hline Double & 1,780 & 38 & 2.1 \\
\hline Triple & 2,176 & 38 & 1.7 \\
\hline Quadruple & 756 & 7 & 0.9 \\
\hline Quintuple + more & 141 & 0 & 0.0 \\
\hline Total & 6,253 & 120 & 1.9 \\
\hline
\end{tabular}

Abbreviation: CABG, coronary artery bypass grafting.
Further improvements in the basic configuration of the registry are necessary to allow a more detailed and particularly a risk-adjusted analysis of the data. However, if significant structural changes of data collection for the registry are conducted, it must be ensured that data compatibility still allows further longitudinal data analysis.

Completeness, validity, and further developments will depend on continued efforts of the GSTCVS in close collaboration with all cardiac surgical units in Germany. This will be of outstanding importance in sense of a contribution for patient safety and to adduce evidence for quality of cardiac surgery in Germany.

Table Con1 Congenital heart surgery with/without ECC

\begin{tabular}{|l|l|l|l|}
\hline Age & $N$ & Deaths & $\%$ \\
\hline (A) Without ECC & 26 & 0 & 0.0 \\
\hline Older than 18 y & 144 & 3 & 2.1 \\
\hline $1-17 \mathrm{y}$ & 685 & 18 & 2.6 \\
\hline Younger than 1 y & 855 & 21 & 2.5 \\
\hline Total A & \multicolumn{4}{l}{} \\
\hline (B) With ECC & 962 & 27 & 2.8 \\
\hline Older than 18 y & 1,712 & 15 & 0.9 \\
\hline $1-17$ y & 2,047 & 67 & 3.3 \\
\hline Younger than 1 y & 4,721 & 109 & 2.3 \\
\hline Total B
\end{tabular}

Abbreviation: ECC, extracorporeal circulation. 
Table Con2 Procedures for congenital heart disease with and without ECC

\begin{tabular}{|l|l|l|l|l|l|l|l|l|l|}
\hline \multirow{2}{*}{ Lesion } & \multicolumn{3}{|c|}{ Age $<\mathbf{1}$ y } & \multicolumn{3}{c|}{ Age 1-17 y } & \multicolumn{3}{c|}{ Age $\geq 18$ y } \\
\cline { 2 - 11 } & $N$ & Deaths & $\%$ & $N$ & Deaths & \% & N & Deaths & $\%$ \\
\hline ASD & 35 & 0 & 0.0 & 252 & 0 & 0.0 & 277 & 7 & 2.5 \\
\hline Complete AV canal & 175 & 4 & 2.3 & 81 & 1 & 1.2 & 17 & 0 & 0.0 \\
\hline VSD & 302 & 2 & 0.7 & 119 & 0 & 0.0 & 29 & 2 & 6.9 \\
\hline Fallot tetralogy & 195 & 2 & 1.0 & 42 & 1 & 2.4 & 3 & 0 & 0.0 \\
\hline DORV & 43 & 0 & 0.0 & 14 & 0 & 0.0 & 1 & 0 & 0.0 \\
\hline TGA & 150 & 0 & 0.0 & 2 & 0 & 0.0 & 0 & 0 & - \\
\hline TGA + VSD & 48 & 3 & 6.3 & 3 & 0 & 0.0 & 0 & 0 & - \\
\hline Truncus arteriosus & 31 & 2 & 6.5 & 5 & 0 & 0.0 & 0 & 0 & - \\
\hline Fontan & 4 & 0 & 0.0 & 246 & 3 & 1.2 & 6 & 1 & 16.7 \\
\hline Norwood & 150 & 22 & 14.7 & 0 & 0 & - & 0 & 0 & - \\
\hline Pulmonary valve & 66 & 1 & 1.5 & 226 & 0 & 0.0 & 71 & 2 & 2.8 \\
\hline $\begin{array}{l}\text { Transcatheter pulmonaly } \\
\text { valve implantation }\end{array}$ & 0 & 0 & - & 14 & 0 & 0.0 & 10 & 0 & 0.0 \\
\hline Aortic valve & & & & & & & & & \\
\hline Ross procedure & 48 & 3 & 6.3 & 195 & 0 & 0.0 & 276 & 3 & 1.1 \\
\hline Mitral valve & 6 & 0 & 0.0 & 27 & 0 & 0.0 & 20 & 0 & 0.0 \\
\hline Tricuspid valve & 41 & 1 & 2.4 & 109 & 3 & 2.8 & 94 & 5 & 5.3 \\
\hline PDA & 76 & 0 & 0.0 & 54 & 2 & 3.7 & 56 & 2 & 3.6 \\
\hline Coarctation & 245 & 5 & 2.0 & 13 & 0 & 0.0 & 0 & 0 & - \\
\hline Transplantation heart & 194 & 2 & 1.0 & 30 & 0 & 0.0 & 8 & 0 & 0.0 \\
\hline Transplantation heart + lung & 2 & 0 & 0.0 & 33 & 0 & 0.0 & 0 & 0 & - \\
\hline Transplantation lung & 0 & 0 & - & 0 & 0 & - & 0 & 0 & - \\
\hline Others & 0 & 0 & - & 12 & 1 & 8.3 & 0 & 0 & - \\
\hline Total & 2,732 & 85 & 3.1 & 1,856 & 18 & 1.0 & 988 & 27 & 2.7 \\
\hline
\end{tabular}

Abbreviations: ASD, atrial septal defect; AV, atrioventricular; DORV, double outlet right ventricle; PDA, patent ductus arteriosus; TGA, transposition at the great arteries; VSD, ventricular septal defect.

Table Mis1 Development of Ross procedures in various age groups

\begin{tabular}{|l|l|l|l|l|l|l|l|l|l|l|}
\hline Autologous aortic valve & $\mathbf{2 0 0 4}$ & $\mathbf{2 0 0 5}$ & $\mathbf{2 0 0 6}$ & $\mathbf{2 0 0 7}$ & $\mathbf{2 0 0 8}$ & $\mathbf{2 0 0 9}$ & $\mathbf{2 0 1 0}$ & $\mathbf{2 0 1 1}$ & $\mathbf{2 0 1 2}$ & $\mathbf{2 0 1 3}$ \\
\hline replacement (Ross procedure) & & & & & & & & & & \\
\hline In patients $\geq 18 \mathrm{y}$ & 250 & 235 & 228 & 261 & 207 & 175 & 184 & 134 & 117 & 107 \\
\hline In patients $<18 \mathrm{y}$ & 50 & 46 & 50 & 34 & 42 & 54 & 43 & 40 & 36 & 33 \\
\hline Total & 300 & 281 & 278 & 295 & 249 & 229 & 227 & 174 & 153 & 140 \\
\hline
\end{tabular}

Table Mis2 Organ transplantation

\begin{tabular}{|l|l|l|l|l|l|l|}
\hline \multirow{2}{*}{ Transplantation } & \multicolumn{3}{|c|}{ With ECC } & \multicolumn{3}{c|}{ Without ECC } \\
\cline { 2 - 7 } & $N$ & Deaths & $\%$ & $N$ & Deaths & \\
\hline Heart & 301 & 41 & 13.6 & & & \\
\hline Heart + lung & 12 & 4 & 33.3 & 15.8 & 209 & 10 \\
\hline Lung & 114 & 18 & 4.8 \\
\hline
\end{tabular}

Abbreviation: ECC, extracorporeal circulation.

Notes: All pediatric transplantations (demonstrated in - Table Con2) are included in this table. Eurotransplant (ET) has reported for the same period 297 heart transplantations (HTx), 2 heart + kidney transplantations, 1 heart + liver transplantations, 12 heart-lung transplantations (HLTx), 320 double lung (DLTx), 38 single lung transplantations (SLTx), 0 lung + kidney transplantations, and 0 lung + liver transplantations. 
Table Mis3 Aortic surgery

\begin{tabular}{|c|c|c|c|c|c|c|}
\hline \multirow[t]{2}{*}{ Aortic surgery ${ }^{a}$} & \multicolumn{3}{|c|}{ With ECC } & \multicolumn{3}{|c|}{ Without ECC } \\
\hline & $N$ & Deaths & $\%$ & $N$ & Deaths & $\%$ \\
\hline Supracoronary ascending aorta replacement & 1,634 & 115 & 7.0 & & & \\
\hline Supracoronary ascending + aortic valve replacement & 1,280 & 62 & 4.8 & & & \\
\hline Infracoronary ascending aorta replacement & & & - & & & \\
\hline Mechanical valve conduit & 490 & 29 & 5.9 & & & \\
\hline Biological valve conduit & 872 & 89 & 10.2 & & & \\
\hline David procedure & 472 & 12 & 2.5 & & & \\
\hline Yacoub procedure & 169 & 2 & 1.2 & & & \\
\hline Other & 292 & 13 & 4.5 & & & \\
\hline Aortic arch replacement ${ }^{\mathrm{b}}$ & 1,680 & 212 & 12.6 & & & \\
\hline Replacement of descending aorta & 69 & 5 & 7.2 & 11 & 2 & 18.2 \\
\hline Thoraco-abdominal aortic replacement & 81 & 13 & 16.0 & 26 & 4 & 15.4 \\
\hline Endostent descending aorta & 6 & 0 & 0.0 & 589 & 37 & 6.3 \\
\hline Total & 7,045 & 552 & 7.8 & 626 & 43 & 6.9 \\
\hline
\end{tabular}

Abbreviations: CABG, coronary artery bypass grafting; ECC, extracorporeal circulation.

Notes: All procedures involving aortic surgery are included in this table. Isolated aortic surgery as well as all possible combined procedures (e.g., additional CABG) are summarized in this category.

aprocedures for abdominal aortic diseases are not included: 549 abdominal procedures and 609 endovascular abdominal stents.

${ }^{b}$ All possible combined procedures are included in this category; the only common denominator is aortic arch surgery.

Table Mis4 Pacemaker and ICD procedures

\begin{tabular}{|c|c|c|c|c|c|c|c|}
\hline \multirow[t]{2}{*}{ Device/category } & \multirow[t]{2}{*}{ Total } & \multirow[t]{2}{*}{ Deaths } & \multirow[t]{2}{*}{$\%$ death } & \multicolumn{2}{|c|}{ With ECC } & \multicolumn{2}{|c|}{ Without ECC } \\
\hline & & & & $N$ & Deaths & $N$ & Deaths \\
\hline Pacemaker total & 14,397 & 104 & 0.7 & 29 & 3 & 14,368 & 101 \\
\hline Implantation & 9,315 & 74 & 0.8 & 4 & 2 & 9,311 & 72 \\
\hline Battery exchange & 2,030 & 7 & 0.3 & 1 & 0 & 2,029 & 7 \\
\hline Revision procedures & 3,052 & 23 & 0.8 & 24 & 1 & 3,028 & 22 \\
\hline ICD total & 9,942 & 82 & 0.8 & 29 & 7 & 9,913 & 75 \\
\hline Implantation & 4,613 & 24 & 0.5 & 0 & 0 & 4,613 & 24 \\
\hline Battery exchange & 2,022 & 2 & 0.1 & 0 & 0 & 2,022 & 2 \\
\hline Revision procedures & 3,307 & 56 & 1.7 & 29 & 7 & 3,278 & 49 \\
\hline Miscellaneous & 1,399 & 7 & 0.5 & 1 & 0 & 1,398 & 7 \\
\hline Total & 25,738 & 193 & 0.7 & 59 & 10 & 25,679 & 183 \\
\hline
\end{tabular}

Table Mis5 Surgical ablation procedures

\begin{tabular}{|l|l|l|}
\hline Energy source & Total & Endocardiac \\
\cline { 2 - 4 } & & $N$ \\
\hline Unipolar radiofrequency & 166 & 116 \\
\hline Unipolar cryoradiofrequency & 421 & 246 \\
\hline Bipolar radiofrequency & 2,348 & 296 \\
\hline Cryothermy & 1,593 & 1,387 \\
\hline Microwave & 13 & 2 \\
\hline Focused ultrasound & 434 & 27 \\
\hline Laser & 0 & 2062 \\
\hline Other & 4 & 0 \\
\hline Total & 4,979 & 407 \\
\hline
\end{tabular}

Notes: All isolated ablation procedures and all possible combination of procedures (e.g., CABG + ablation) are included. Total of $n=379$ procedures are not specified with regard to endocardiac/epicardiac ablation. 


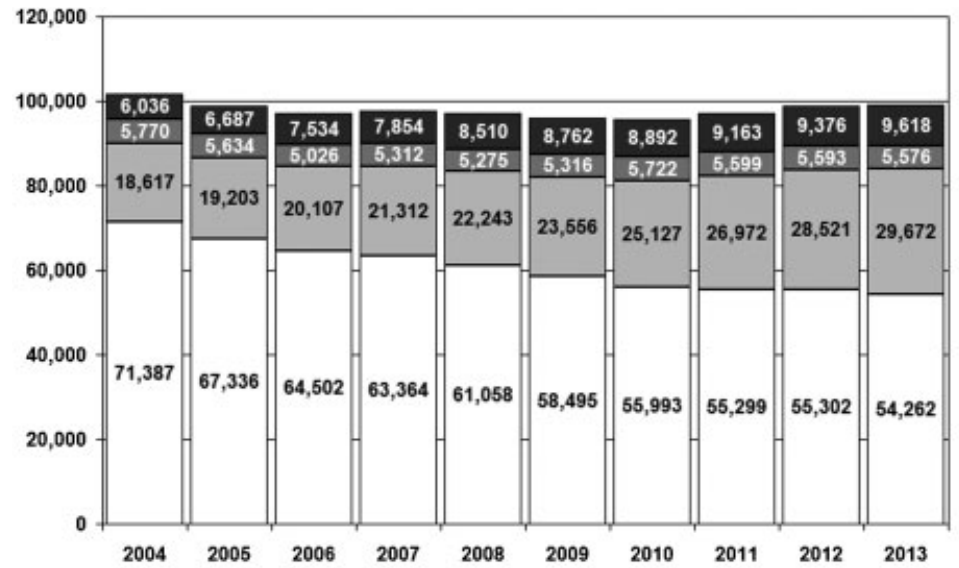

Miscellaneous procedures

a Surgery for CHD

口 Heart valve procedures

$\square C A B G$ and combinations

Fig. 1 Cardiac surgery in Germany from 2004 to 2013. (1) Coronary artery bypass grafting (CABG) and combinations: all types of isolated coronary surgery with or without extracorporeal circulation (ECC) and any combined procedure. (2) Valve procedures: all types of isolated heart valve surgery; heart valve procedures in combination with aortic surgery are summarized in the miscellaneous group. (3) Congenital heart surgery: all procedures with or without ECC; atrial septal defect repair in adults in combination with CABG or heart valve surgery are summarized in the CABG or heart valve surgery group. (4) Miscellaneous procedures: all other types of procedures with ECC.

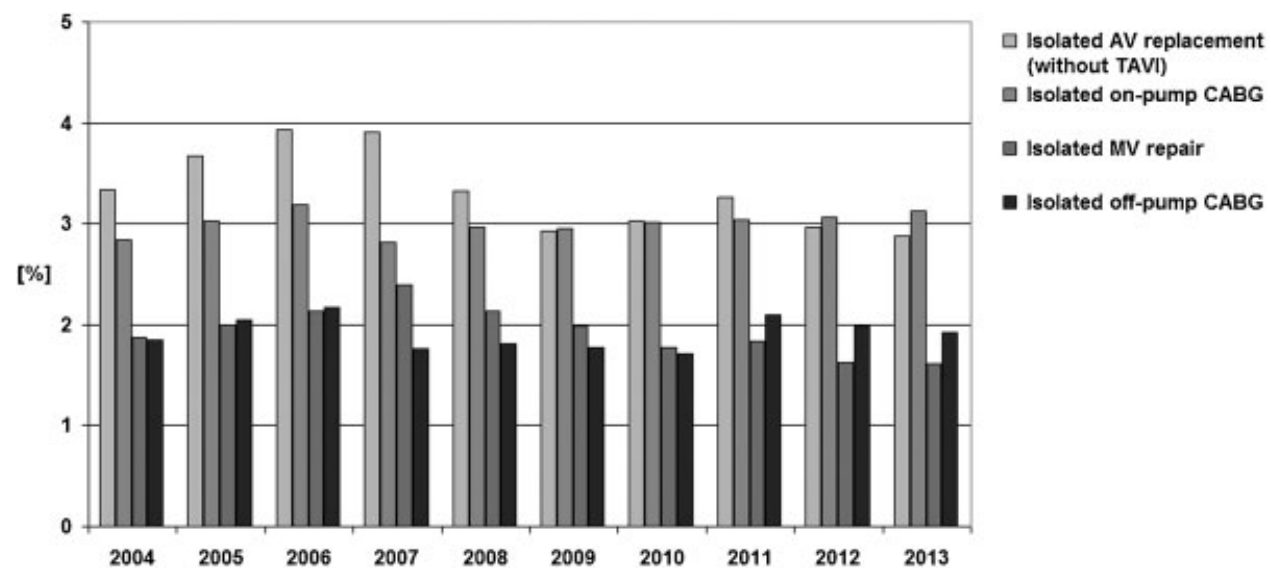

Fig. 2 Unadjusted mortality for selected procedures 2004 to 2013.

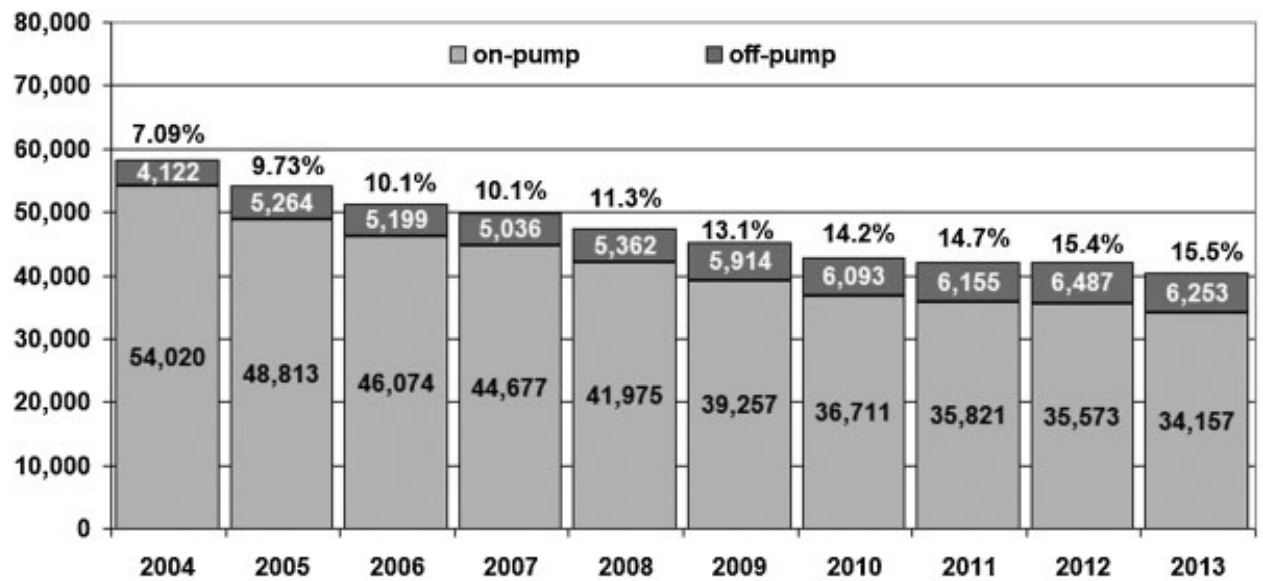

Fig. 3 Isolated coronary artery bypass grafting. The number of coronary artery bypass procedures declined since the year 2004 while the percentage of off-pump procedures slightly increased. 


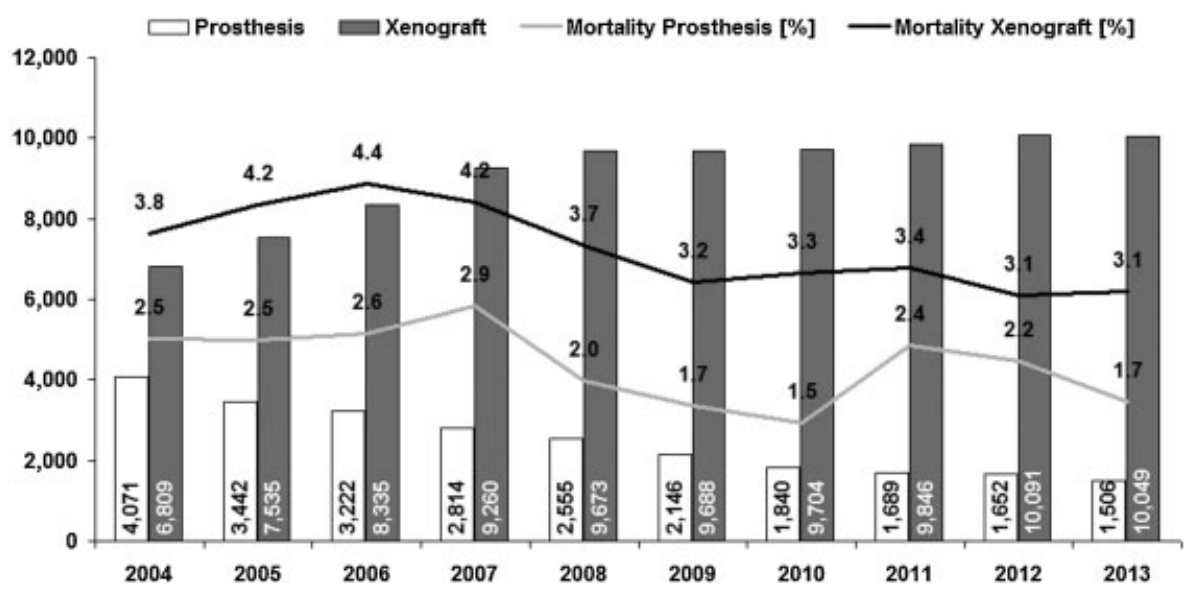

Fig. 4 Isolated aortic valve replacement from 2004 to 2013 in Germany. The use of xenografts continuously increased till 2008 and remained almost the same since then. Aortic valve replacement (AVR) using mechanical prosthesis decreased over the past 10 years. The sustained difference in mortality seems to be related to the difference in age pattern. (Ross, homograft implantations, and transapical or transvascular aortic valve implantation [TAVI] are excluded.)

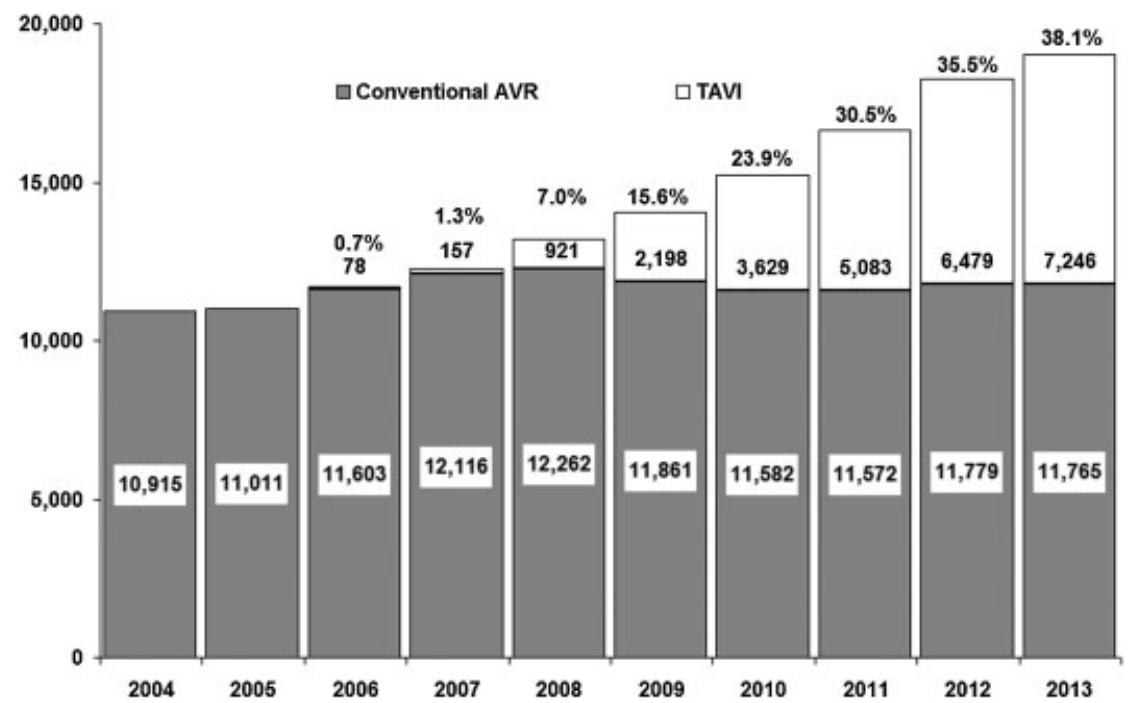

Fig. 5 Isolated AVR and transapical or transvascular aortic valve implantation (TAVI). The figure shows almost unvaried quantity of AVR and an obvious increase of TAVI. In 2013, 38.1\% of reported isolated aortic valve procedures were performed by transvascular or transapical implantation.

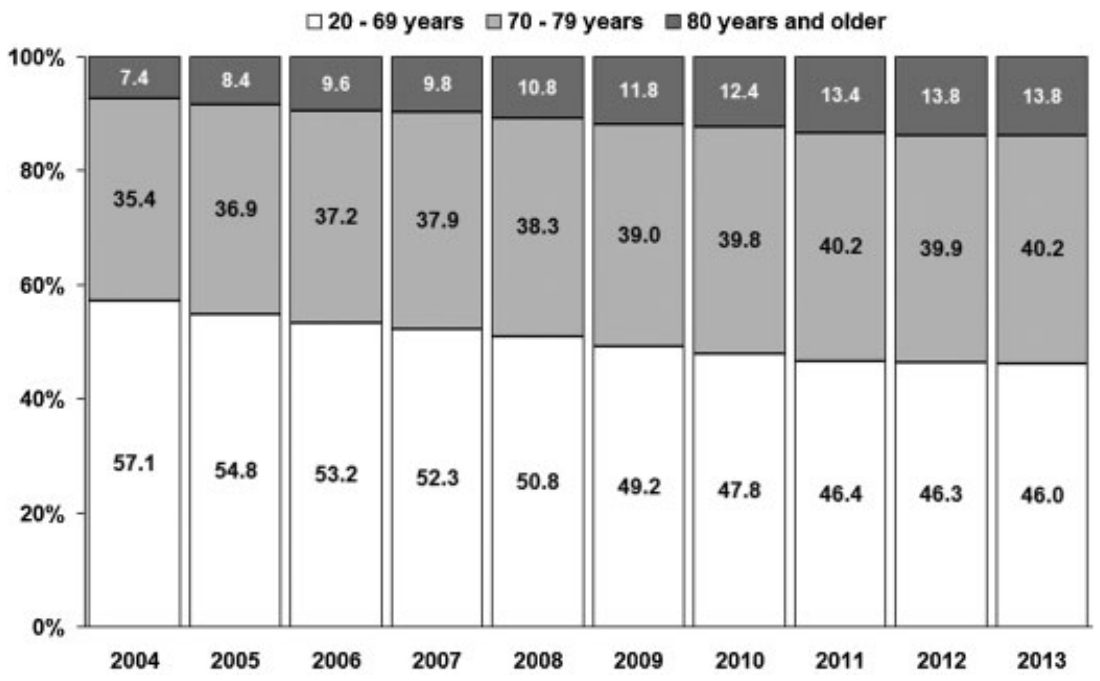

Fig. 6 Age distribution of cardiac procedures since 2004. Currently, 54.0\% of the patients are at least 70 years (patients $<20$ years and ICD/ pacemaker procedures are excluded). 
$\square$ Emergency

2004

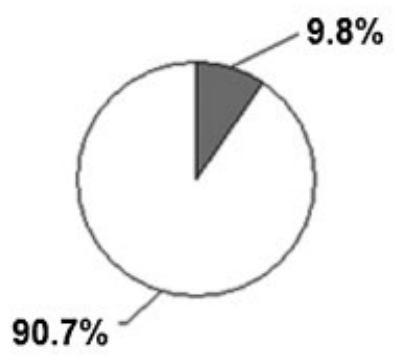

Elective / urgent

2013

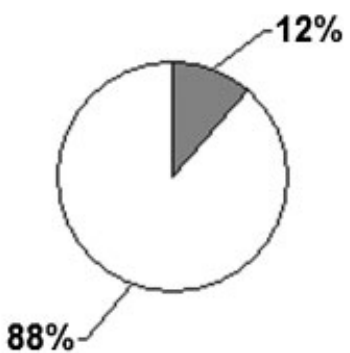

Fig. 7 Urgency 2004 and 2013.

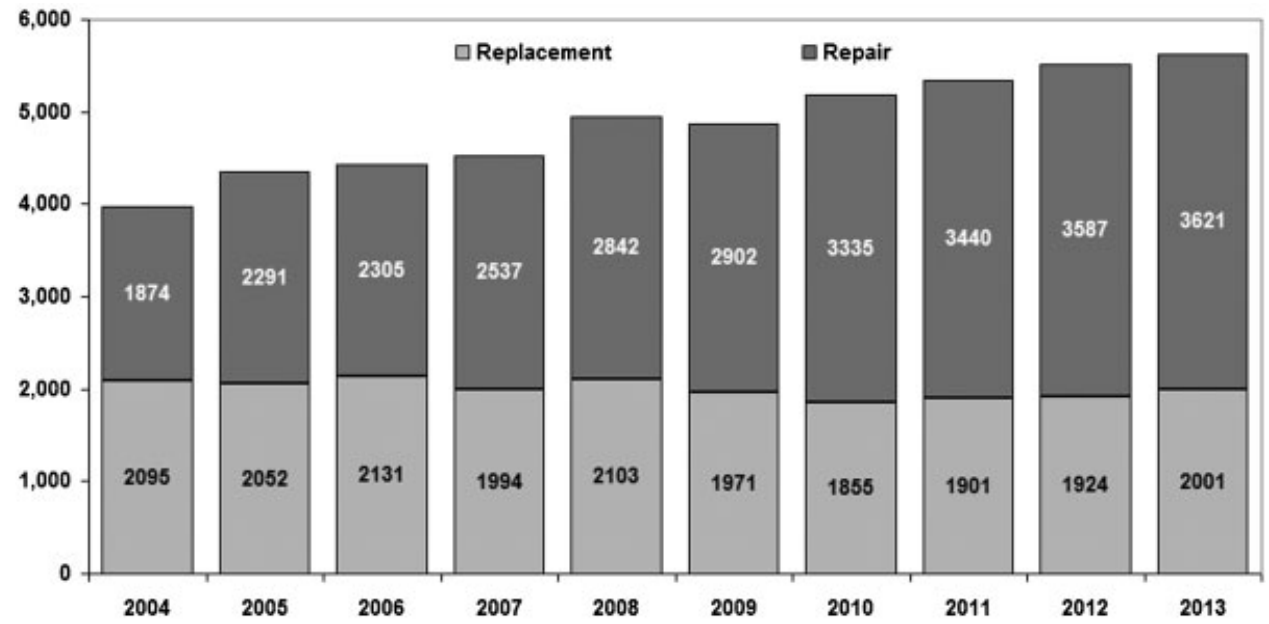

Fig. 8 Isolated mitral valve surgery over the past 10 years. In $65.8 \%$ mitral valve (MV) repairs and in $34.2 \%$ MV replacements were performed. In 1994 , the rate of reconstruction just reached $21 \%$.

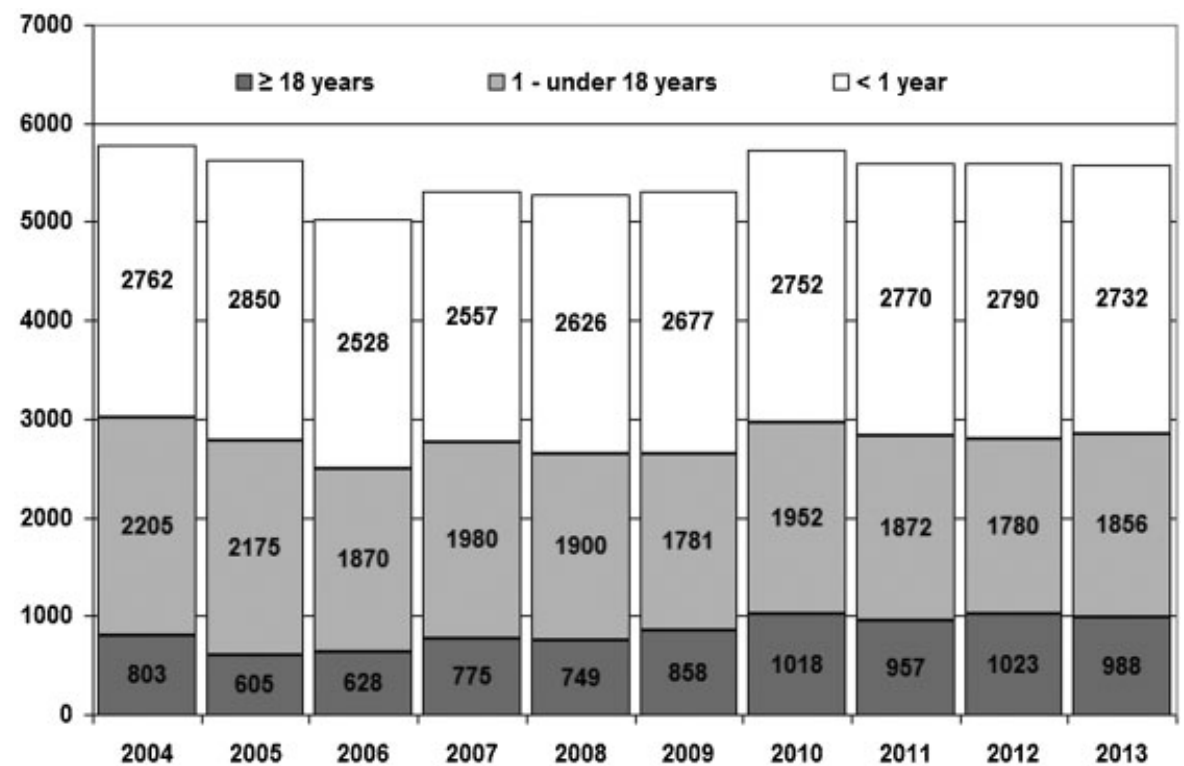

Fig. 9 Age distribution for congenital heart surgery in Germany over the past 10 years. No relevant changes can be observed. However, there may be a bias since not all relevant procedures can be allocated clearly to the congenital heart disease group in patients older than 18 years (e.g., aortic valve disease). 


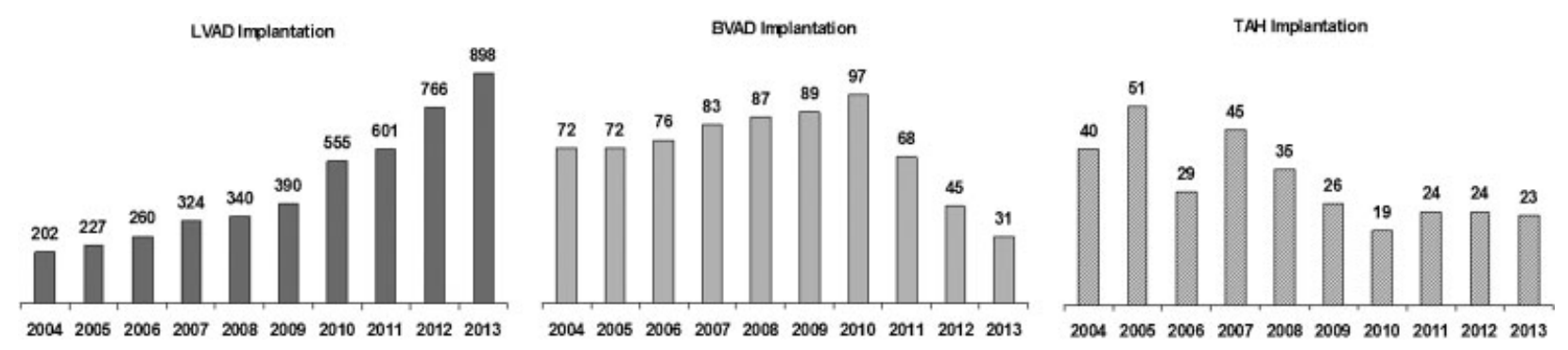

Fig. 10 Development of mechanical circulatory support in Germany over the past 10 years. Significant increase of left ventricular assist device (LVAD) implantations. However, the number of implanted paracorporeal biventricular assist devices (BVAD) dropped down by $68 \%$ during the past 4 years. The number of total artificial heart implantations (TAH) remained on the same low level.

\section{Acknowledgments}

On behalf of the German Society for Thoracic and Cardiovascular Surgery, the authors thank the chairmen and their coworkers of all cardiac surgery units in Germany for their continued cooperation and support to realize this registry.

\section{References}

1 Kalmar P, Irrgang E. Cardiac surgery in the Federal Republic of Germany during 1989. A report by the German Society for Thoracic and Cardiovascular Surgery. Thorac Cardiovasc Surg 1990;38(3):198-200

2 Kalmar P, Irrgang E. Cardiac surgery in the Federal Republic of Germany during 1990. A report by the German Society for Thoracic and Cardiovascular Surgery. Thorac Cardiovasc Surg 1991;39(3):167-169

3 Kalmar P, Irrgang E. Cardiac surgery in Germany during 1991. A report by the German Society for Thoracic and Cardiovascular Surgery. Thorac Cardiovasc Surg 1992;40(3):163-165

4 Kalmar P, Irrgang E. Cardiac surgery in Germany during 1992. A report by the German Society for Thoracic and Cardiovascular Surgery. Thorac Cardiovasc Surg 1993;41(3):202-204

5 Kalmar P, Irrgang E. Cardiac surgery in Germany during 1993. A report by the German Society for Thoracic and Cardiovascular Surgery. Thorac Cardiovasc Surg 1994;42(3):194-196

6 Kalmar P, Irrgang E. Cardiac surgery in Germany during 1994. A report by the German Society for Thoracic and Cardiovascular Surgery. Thorac Cardiovasc Surg 1995;43(3):181-183

7 Kalmár P, Irrgang E. Cardiac surgery in Germany during 1995. A report by the German Society for Thoracic and Cardiovascular Surgery. Thorac Cardiovasc Surg 1996;44(3):161-164

8 Kalmár P, Irrgang E. Cardiac surgery in Germany during 1996. A report by the German Society for Thoracic and Cardiovascular Surgery. Thorac Cardiovasc Surg 1997;45(3):134-137

9 Kalmár P, Irrgang E. Cardiac surgery in Germany during 1997. A report by the German Society for Thoracic and Cardiovascular Surgery. Thorac Cardiovasc Surg 1998;46(5):307-310

10 Kalmàr P, Irrgang E. Cardiac surgery in Germany during 1998. A report by the German Society for Thoracic and Cardiovascular Surgery. Thorac Cardiovasc Surg 1999;47(4):260-263

11 Kalmár P, Irrgang E. Cardiac surgery in Germany during 1999. Thorac Cardiovasc Surg 2000;48(4):XXVII-XXX

12 Kalmar P, Irrgang E. Cardiac surgery in Germany during 2000. Thorac Cardiovasc Surg 2001;49(5):33-38

13 Kalmár P, Irrgang E. Cardiac surgery in Germany during 2001: a report by the German Society for Thoracic and Cardiovascular Surgery. Thorac Cardiovasc Surg 2002;50(6):30-35
14 Kalmár P, Irrgang E; German Society for Thoracic and Cardiovascular Surgery. Cardiac surgery in Germany during 2002: a report by German Society for Thoracic and Cardiovascular Surgery. Thorac Cardiovasc Surg 2003;51(5):25-29

15 Kalmár P, Irrgang E; German Society for Thoracic and Cardiovascular Surgery. Cardiac surgery in Germany during 2003: a report by the German Society for Thoracic and Cardiovascular Surgery. Thorac Cardiovasc Surg 2004;52(5):312-317

16 Gummert JF, Funkat A, Krian A. Cardiac surgery in Germany during 2004: a report on behalf of the German Society for Thoracic and Cardiovascular Surgery. Thorac Cardiovasc Surg 2005;53(6): 391-399

17 Gummert JF, Funkat A, Beckmann A, Hekmat K, Ernst M, Krian A. Cardiac surgery in Germany during 2005: a report on behalf of the German Society for Thoracic and Cardiovascular Surgery. Thorac Cardiovasc Surg 2006;54(5):362-371

18 Gummert JF, Funkat A, Beckmann A, et al. Cardiac surgery in Germany during 2006: a report on behalf of the German Society for Thoracic and Cardiovascular Surgery. Thorac Cardiovasc Surg 2007;55(6):343-350

19 Gummert JF, Funkat A, Beckmann A, et al; German Society for Thoracic and Cardiovascular Surgery. Cardiac surgery in Germany during 2007: a report on behalf of the German Society for Thoracic and Cardiovascular Surgery. Thorac Cardiovasc Surg 2008;56(6): 328-336

20 Gummert JF, Funkat A, Beckmann A, et al. Cardiac surgery in Germany during 2008. A report on behalf of the German Society for Thoracic and Cardiovascular Surgery. Thorac Cardiovasc Surg 2009;57(6):315-323

21 Gummert JF, Funkat A, Beckmann A, et al. Cardiac surgery in Germany during 2009. A report on behalf of the German Society for Thoracic and Cardiovascular Surgery. Thorac Cardiovasc Surg 2010;58(7):379-386

22 Gummert JF, Funkat AK, Beckmann A, et al. Cardiac surgery in Germany during 2010: a report on behalf of the German Society for Thoracic and Cardiovascular Surgery. Thorac Cardiovasc Surg 2011;59(5):259-267

23 Funkat AK, Beckmann A, Lewandowski J, et al. Cardiac surgery in Germany during 2011: a report on behalf of the German Society for Thoracic and Cardiovascular Surgery. Thorac Cardiovasc Surg 2012;60(6):371-382

24 Beckmann A, Funkat AK, Lewandowski J, et al. Cardiac surgery in Germany during 2012: a report on behalf of the German Society for Thoracic and Cardiovascular Surgery. Thorac Cardiovasc Surg 2014;62(1):5-17

25 Gammie JS, Zhao Y, Peterson ED, O’Brien SM, Rankin JS, Griffith BPJ. J. Maxwell Chamberlain Memorial Paper for adult cardiac surgery. Lessinvasive mitral valve operations: trends and outcomes from the 
Society of Thoracic Surgeons Adult Cardiac Surgery Database. Ann Thorac Surg 2010;90(5):1401-1408, e1, discussion 1408-1410

26 Joint Task Force on the Management of Valvular Heart Disease of the European Society of Cardiology (ESC); European Association for Cardio-Thoracic Surgery (EACTS),Vahanian A, Alfieri O, Andreotti F, Antunes MJ, et al. Guidelines on the management of valvular heart disease (version 2012). EurHeart J 2012;33(19):2451-2496

27 Beckmann A, Hamm C, Figulla HR, et al; GARY Executive Board. The German Aortic Valve Registry (GARY): a nationwide registry for patients undergoing invasive therapy for severe aortic valve stenosis. Thorac Cardiovasc Surg 2012;60(5): 319-325

28 Hamm CW, Möllmann H, Holzhey D, et al; for the GARY-Executive Board. The German Aortic Valve Registry (GARY): in-hospital outcome. Eur Heart J 2013

29 Herbert MA, Prince SL, Williams JL, Magee MJ, Mack MJ. Are unaudited records from an outcomes registry database accurate? Ann Thorac Surg 2004;77(6):1960-1964, discussion 1964-1965 